\title{
Up-regulated Endonuclease Regnase-1 Suppresses Osteoarthritis by Forming Negative Feedback Loop of Catabolic Signaling in Chondrocytes
}

Jeong-In Yang

Gwangju Institute of Science and Technology

Jang-Soo Chun ( $\sim$ jschun@gist.ac.kr)

Gwangju Institute of Science and Technology https://orcid.org/0000-0003-2910-181X

\section{Research article}

Keywords: Regnase-1, Chondrocytes, Cartilage, Osteoarthritis, Matrix-degrading enzymes

Posted Date: October 29th, 2020

DOI: https://doi.org/10.21203/rs.3.rs-97816/v1

License: (c) (1) This work is licensed under a Creative Commons Attribution 4.0 International License.

Read Full License

Version of Record: A version of this preprint was published at Arthritis Research \& Therapy on April 14th, 2021. See the published version at https://doi.org/10.1186/s13075-021-02485-z. 


\section{Abstract}

Background: Ribonucleases (RNases) play central roles in the post-transcriptional regulation of mRNA stability. Our preliminary results revealed that the endonuclease Regnase- 1 is specifically up-regulated in osteoarthritic chondrocytes. We herein explored the possible functions and regulatory mechanisms of Regnase- 1 in a mouse model of osteoarthritis (OA).

Methods: The expression and target genes of Regnase- 1 were identified by microarray analysis in primary-culture mouse articular chondrocytes. Experimental OA in mice was induced by destabilization of the medial meniscus (DMM). The function of Regnase-1 in DMM-induced post-traumatic OA mice was examined by adenovirus-mediated overexpression or knockdown in knee joint tissues, and also by using Regnase-1 heterozygous knockout mice $\left(Z c 3 h 12 a^{+/}\right)$.

Results: Among the RNases, Regnase- 1 was exclusively up-regulated in chondrocytes stimulated with OAassociated catabolic factors. Adenovirus-mediated overexpression or knockdown of Regnase-1 alone in joint tissues did not cause OA-like changes. However, overexpression of Regnase- 1 in joint tissues significantly ameliorated DMM-induced post-traumatic OA cartilage destruction, whereas knockdown or genetic ablation of Regnase-1 exacerbated DMM-induced cartilage destruction. Mechanistic studies showed that Regnase- 1 appears to suppress cartilage destruction by modulating the expression of matrix-degrading enzymes in chondrocytes.

Conclusion: Our results collectively suggest that up-regulated Regnase-1 in OA chondrocytes may function as a chondro-protective effector molecule during OA pathogenesis by forming negative feedback loop of catabolic signaling such as expression of matrix-degrading enzymes in OA chondrocytes.

\section{Background}

Osteoarthritis $(\mathrm{OA})$ is a whole-joint disease characterized by cartilage destruction, synovial inflammation, osteophyte formation, subchondral bone remodeling, etc [1]. Among these manifestations of OA, progressive articular cartilage degradation is a hallmark of $O A$. This degradation is primarily regulated by chondrocytes through the production of matrix-degrading enzymes and/or the down-regulation of cartilage extracellular matrix (ECM) molecules [2]. Among the matrix-degrading enzymes, matrix metalloproteinase 3 (MMP3), MMP13, and ADAMTS5 are known to play important roles in OA cartilage destruction [3-5]. In chondrocytes, the expression levels of these molecules are regulated by extracellular catabolic regulators, such as the pro-inflammatory cytokine, interleukin (IL)-1 [6]. We previously identified cellular catabolic mediators in chondrocytes, including the transcription factor, hypoxiainducible factor (HIF)-2a [7], the zinc importer, ZIP8 [8], and the cholesterol hydroxylase, $\mathrm{CH} 25 \mathrm{H}$ [9]. These cellular mediators exert catabolic functions by up-regulating matrix-degrading enzymes and/or downregulating ECM molecules in chondrocytes.

The expression of OA-related catabolic and anabolic factors in chondrocytes can be regulated at multiple checkpoints, including transcriptional and post-transcriptional levels. The ribonucleases (RNases) 
comprise a broad class of RNA-degrading enzymes that play central roles in the post-transcriptional regulation of mRNA stability [10]. However, the roles of specific ribonucleases and their targets in OA pathogenesis have not yet been clearly elucidated. Here, we initially used a microarray analysis to screen for RNases whose expression were modulated in chondrocytes upon stimulation with OA-associated catabolic factors, such as IL-1 $\beta$ treatment, ZIP8 overexpression, or HIF-2a overexpression [6-8]. Among the examined RNases, our initial screening analysis revealed that Regnase-1 (a zinc finger CCCH-type containing $12 \mathrm{~A}$ encoded by $Z c 3 h 12 a$ in mouse) was exclusively up-regulated in chondrocytes stimulated with OA-associated catabolic factors. Regnase-1, which is also known as MCPIP1 (monocyte chemotactic protein-induced protein 1), is an endonuclease that destabilizes various target mRNAs by recognizing a stem-loop structure within their $3^{\prime}$-UTRs $[11,12]$. Regnase- 1 is known to play diverse functions in different cell types, such as mitigating inflammation by down-regulating the IL-6 and IL-12B mRNAs in monocytes [11]; restricting T cell activation by targeting the c-Rel, Ox40, and IL-2 mRNAs in T cells $[13,14]$; and promoting cancer cell apoptosis by regulating mRNAs of Bcl2L1, Bcl2A1, RelB, and Bcl3 [15].

One of the known Regnase- 1 target in chondrocytes is IL-6 [16, 17], which plays an important role in OA pathogenesis $[18,19]$. However, the role of Regnase-1 in OA pathogenesis in vivo has not been investigated to date. Thus, we herein explored the possible functions and regulatory mechanisms of Regnase-1 in mouse models of post-traumatic OA. Our gain-of-function (adenovirus-mediated overexpression in joint tissues) and loss-of-function $\left(Z c 3 h 12 a^{+/-}\right)$approaches clearly indicated that upregulated Regnase- 1 in OA chondrocytes exerts chondro-protective functions at least partly by modulating the expression levels of matrix-degrading enzymes (MMP3, MMP13, and ADAMTS5) in articular chondrocytes.

\section{Methods}

\section{Mice and experimental RA}

C57BL/6J mice were used for the experimental OA studies. C57BL/6J-background Regnase-1-KO mice (68-bp deletion in exon 2) were generated by ToolGen, Inc. (Seoul, Korea). Because homozygous KO mice $\left(Z c 3 h 12 a^{-/-}\right)$die shortly after weaning [20], we used heterozygous $Z c 3 h 12 a^{+/-}$mice for our experimental OA studies. All experiments were approved by the Animal Care and Use Committee of Gwangju Institute of Science and Technology. Post-traumatic OA was induced in 12-week-old male mice by destabilization of the medial meniscus (DMM), and the mice were sacrificed at the indicated weeks after the surgery [21]. Experimental OA was also induced by IA injection (once weekly for 3 weeks) of adenovirus $\left[1 \times 10^{9}\right.$ plaque-forming units (PFUs) in a total volume of $10 \mu \mathrm{l}$ ] expressing Regnase-1 (Ad-Regnase-1), SAA3 (AdSAA3), ZIP8 (Ad-ZIP8), or HIF-2a (Ad-HIF-2a). Mice were sacrificed 3 weeks after the first injection of adenovirus [7-9]. When performing IA injection after DMM surgery, the first injection was started at 10 days after surgery, and IA injections were performed weekly for a total of three injections. The mice were sacrificed at the indicated weeks after the surgery. 


\section{Histological analysis}

Mouse knee joint samples were fixed with $4 \%$ paraformaldehyde, decalcified in $0.5 \mathrm{M}$ EDTA, embedded in paraffin, and sectioned frontally at 5 - $\mu \mathrm{m}$ thickness. Sections were deparaffinized in xylene, hydrated with graded ethanol, and stained with Safranin-0 [8, 9]. Cartilage destruction was scored by four observers under blinded conditions using the OARSI scoring system (grade 0-6) [22]. Synovitis was determined by Safranin-0 and hematoxylin staining, and synovial inflammation (grade $0-3$ ) was scored [23]. Osteophytes were identified by Safranin-0 staining, and osteophyte size was measured with an Aperio ImageScope Version 12.4.0 (Leica Biosystems) [4]. We measured the thickness of the subchondral bone plate (SBP) using an Aperio ImageScope to assess subchondral bone sclerosis [24]. Skeletons of E18.5 whole-mouse embryos were stained with Alcian blue and Alizarin red [8, 9]. The expression of GFP in cartilage tissues of mice IA-injected with GFP-tagged Ad-Regnase-1 was detected by standard fluorescence microscopy

\section{Primary culture of mouse articular chondrocytes}

Mouse articular chondrocytes were isolated from the femoral condyles and tibial plateaus of 5-day-old WT or $Z c 3 h 12 a^{-/-}$mice by $0.2 \%$ collagenase digestion [25]. The cells were maintained as a monolayer in Dulbecco's modified Eagle's medium (DMEM; Gibco) supplemented with $10 \%$ fetal bovine serum and antibiotics (penicillin $\mathrm{G}$ and streptomycin). On culture day 2, cells were infected with the indicated $\mathrm{MOI}$ (multiplicity of infection) of empty adenovirus (Ad-C), Ad-Regnase-1, Ad-shRegnase-1, Ad-SAA3, Ad-HIF$2 a$, or Ad-ZIP8 or treated with IL-1 $\beta$ as indicated in each experiment.

\section{Western blotting}

Total cell lysates were prepared in lysis buffer [150 mM NaCl, 1\% NP-40, $50 \mathrm{mM}$ Tris, $0.2 \%$ sodium dodecyl sulfate (SDS), $5 \mathrm{mM} \mathrm{NaF}$ ] containing a cocktail of protease inhibitors and phosphatase inhibitors (Roche). Rabbit polyclonal anti-Regnase-1 (Novus Biologicals) and anti-Lamin B (Santa Cruz Biotech) antibodies were used for Western blotting.

\section{Microarray analysis}

Our microarray data from chondrocytes stimulated with IL-1 $\beta$ treatment or those overexpressing HIF-2a (Ad-HIF-2a infection) or ZIP8 (Ad-ZIP8 infection) were previously deposited to the Gene Expression Omnibus under accession codes GSE104794 (HIF-2a), GSE104795 (ZIP8), and GSE104793 (IL-1ß). We also performed microarray analysis in chondrocytes infected with $800 \mathrm{MOI}$ of Ad-Regnase-1 or AdshRegnase-1 for 36 hours. Briefly, total RNA was extracted from mouse articular chondrocytes using a Purelink RNA mini kit (Ambion) and analyzed using Affymetrix Gene Chip arrays (Affymetrix GeneChip Mouse Gene 2.0 ST Array) in accordance with the Affymetrix protocol (Macrogen Inc.). The probe signals in the raw data were normalized with respect to the RMA (Robust Multi-array Average) for each separate data set (infection of Ad-C, Ad-Regnase-1, Ad-shC, or Ad-shRegnase-1). These microarray data were deposited to the Gene Expression Omnibus under accession codes GSE153179 (for Ad-Regnase-1 and Ad-shRegnase-1). 


\section{Reverse transcription-polymerase chain reaction (RT-PCR) and quantitative RT-PCR (qRT-PCR) analysis}

Total RNA was extracted from primary-culture chondrocytes using the TRI reagent (Molecular Research Center, Inc.). Total RNA was reverse transcribed, and the resulting CDNA was PCR amplified using the PCR primers and experimental conditions summarized in Supplementary Table 1. qRT-PCR was performed using an iCycler thermal cycler (Bio-Rad) and SYBR premixExTaq. Transcript levels were normalized with respect to those of $\beta$-actin and expressed as fold changes relative to the control.

\section{Luciferase assay}

The SAA3-3'-UTR reporter plasmid, which contained the 5'-AATAAATACTTGTGAAATGCA-3' sequence of 3 '-UTR of SAA3, was purchased from GeneCopoeia. Primary-culture mouse chondrocytes were pretreated with hyaluronidase type I-S (Sigma) for 3 hours in serum-free DMEM, and transfected by incubation for 6 hours with SAA3-3'-UTR reporter vector $(0.05 \mu \mathrm{g})$ and Lipofectamine 2000 , as described by the manufacturer. The cells were co-transfected with $0.05 \mu \mathrm{g}$ of empty vector (Origene), WT-Regnase-1 expression vector (Origene), or D141N Regnase-1 (which lacks RNase activity) [13]. The cells were harvested at 24 hours after treatment, and firefly luciferase and Renilla luciferase activities were measured using a Dual-Luciferase Assay System (Promega).

\section{Statistical analysis}

For statistical comparison of experimental groups, the data were analyzed by the Shapiro-Wilk test for normality and Levene's test for homogeneity of variance. Two groups of non-parametric data based on the ordinal grading systems (OARSI grade, synovitis, osteophyte maturity) were compared using the Mann-Whitney $U$ test, whereas the Kruskal-Wallis test was used to compare multi-groups of nonparametric data. We also used the Mann-Whitney $U$ test for the direct comparison of pairs of groups among the multi-groups of non-parametric data. Parametric data collected from two independent experimental groups were compared by two-tailed $t$-test. For comparisons of three or more groups of parametric data, one-way analysis of variance (ANOVA) with Bonferroni's post-hoc test was used. Each $n$ number indicates the number of biologically independent samples or mice per group. Significance was accepted at the 0.05 level of probability $(p<0.05)$. Each bar represents the s.e.m. for parametric data and the calculated $95 \%$ confidence interval $(\mathrm{Cl})$ for nonparametric data.

\section{Results}

\section{OA-associated catabolic factors up-regulate Regnase-1 in mouse articular chondrocytes}

To identify RNases that may be associated with OA pathogenesis, we analyzed the expression levels of RNases (both endonucleases and exonucleases) in primary-culture articular chondrocytes stimulated with OA-associated catabolic factors, including a pro-inflammatory cytokine (IL-1 $\beta$ ) [6] and adenovirusmediated overexpression of HIF-2a (Ad-HIF-2a) [7] or ZIP8 (Ad-ZIP8) [8]. Our microarray analysis revealed 
that Regnase-1 (encoded by Zc3h12a) was exclusively up-regulated in chondrocytes stimulated with the tested catabolic regulators (Fig. 1A, Supplementary Table 2). We further characterized the expression of Regnase- 1 in primary-culture chondrocytes. Unstimulated chondrocytes exhibited various mRNA levels of ZC3H12 family members (Fig. 1B), whereas our RT-PCR (Supplementary Fig. 1) and quantitative RT-PCR (qRT-PCR) (Fig. 1B) analyses revealed that Regnase-1 was exclusively up-regulated by the OA-associated catabolic factors (i.e., IL-1 $\beta$ treatment or infection of Ad-HIF-2 $a$ or Ad-ZIP8). Regnase-1 protein levels were also increased by Ad-Regnase-1 infection or IL-1 $\beta$ treatment in primary-culture chondrocytes (Fig. 1C).

\section{Overexpression of Regnase-1 in joint tissues ameliorates post-traumatic OA cartilage destruction in mice}

Because the above results suggest that up-regulated Regnase- 1 in OA-like chondrocytes may play a role in OA pathogenesis, we examined the functions of Regnase-1 in OA development by overexpressing it in mouse knee joint tissues via intra-articular (IA) injection of GFP-tagged Ad-Regnase-1. We previously reported that an adenoviral system can be used to effectively deliver transgenes into joint tissues [7-9]. Consistent with this, detection of GFP fluorescence confirmed the overexpression of Regnase- 1 in cartilage tissue (Fig. 2A). However, this overexpression of Regnase-1 did not cause any OA-like change in the cartilage or synovium of the joint tissues (Fig. 2B). Consistently, Regnase-1 overexpression in primaryculture chondrocytes did not affect mRNA levels of matrix-degrading enzymes (MMP3, MMP13, and ADAMTS5), cartilage ECM molecules (type II collagen and aggrecan), or cellular catabolic regulators (HIF$2 a$ and ZIP8) (Fig. 2C).

We further examined the effects of Regnase-1 overexpression in knee joint tissues under DMM-induced post-traumatic OA. Unlikely to the effects of Regnase- 1 overexpression alone, adenoviral overexpression of Regnase-1 in joint tissues in DMM-operated mice significantly suppressed post-traumatic OA cartilage destruction (Fig. 2D and E). Other examined manifestations of OA, such as osteophyte formation and thickness of the subchondral bone plate (SBP), also tended to be reduced by Regnase- 1 overexpression, but the differences did not reach the level of statistical significance (Fig. 2D and E). These findings suggest that overexpression of Regnase- 1 alone does not cause OA pathogenesis; instead, up-regulated Regnase-1 in OA chondrocytes appears to play a protective role in DMM-induced post-traumatic cartilage destruction in mice.

\section{Knockdown of Regnase-1 exacerbates post-traumatic OA cartilage destruction.}

To further elucidate the functions of Regnase-1 in OA pathogenesis, we generated Zc3h12a KO mice or by knocking down Regnase-1 in whole-joint tissues via IA injection of an adenovirus expressing shRNA against Regnase-1 (Ad-shRegnase-1). IA injection of Ad-shRegnase-1 alone did not cause cartilage damage (Supplementary Fig. 2A). Additionally, Regnase-1 knockdown in primary-culture chondrocytes did not affect the expression levels of matrix-degrading enzymes or cartilage ECM molecules (Supplementary Fig. 2B). However, knock-down of Regnase-1 via IA injection of Ad-shRegnase-1 in DMM-operated mice significantly enhanced DMM-induced OA manifestations such as cartilage destruction, osteophyte formation, and thickening of the SBP plate (Fig. 3A and B). This is consistent with the idea that up- 
regulated Regnase-1 in OA chondrocytes has chondro-protective effects in DMM-induced OA pathogenesis.

We further validated the functions of Regnase- 1 in OA pathogenesis by using a Regnase- 1 KO mouse generated by deleting 68 bp from exon 2 of the Zc3h12a gene (Supplementary Fig. 3A and B). Because

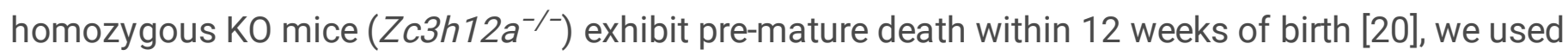

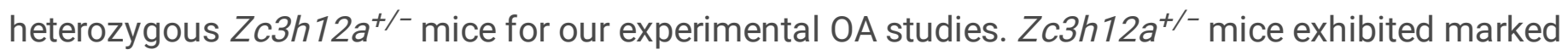
decreases in the mRNA levels of Regnase- 1 in the chondrocytes of their cartilage tissues, but had normal skeletal development in E18.5 embryos (Supplementary Fig. 3C). Compared with WT littermates, $Z c 3 h 12 a^{+/-}$mice exhibited significant enhancement of DMM-induced cartilage erosion but no significant change in osteophyte formation or SBP thickness (Fig. 3C and D). These results additionally indicate that up-regulated Regnase-1 exerts protective functions in DMM-induced post-traumatic OA cartilage destruction in mice.

\section{Regnase-1 modulates the expression levels of matrix-degrading enzymes in chondrocytes}

To elucidate possible mechanisms underlying the protective effects of Regnase-1, we examined whether Regnase-1 modulates the expression levels of matrix-degrading enzymes and cartilage ECM molecules in chondrocytes. For this purpose, Regnase- 1 was overexpressed in primary-culture chondrocytes via AdRegnase-1 infection, and the chondrocytes were stimulated with IL-1 $\beta$. Among the examined molecules, the mRNA level of MMP3 (but not those of MMP13, ADAMTS5, or cartilage ECM molecules) was slightly but significantly decreased by the overexpression of Regnase-1 in IL-1 $\beta$-treated chondrocytes (Fig. 4A and B). Similarly, the HIF-2a-induced increase in the mRNA level of MMP3 was also slightly but significantly decreased by Regnase-1 overexpression, whereas the mRNA levels of the other examined catabolic and anabolic factors were not modulated (Fig. 4C and D).

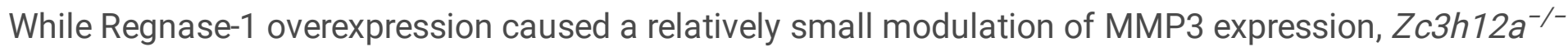
chondrocytes exhibited a more dramatic modulation of the expression levels of matrix-degrading enzymes: Zc3h12a $a^{-/-}$chondrocytes exhibited significantly increased mRNA levels of MMP3, MMP13, and ADAMTS5 under IL-1 $\beta$ treatment, compared to IL-1 $\beta$-treated WT chondrocytes (Fig. 5A). The upregulations of MMP3, MMP13, and ADAMTS5 in HIF-2a-overexpressing chondrocytes were also

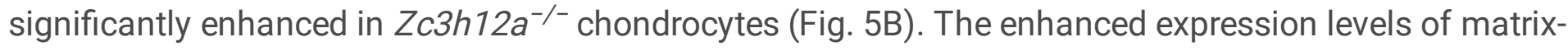
degrading enzymes in $Z c 3 h 12 a^{-/-}$chondrocytes were well consistent with the enhanced OA cartilage destruction seen in DMM-operated Zc3h12a-/- mice.

Although overexpression or knockdown of Regnase-1 alone did not modulate expression of matrixdegrading enzymes in chondrocytes (Fig. 2C, Supplementary Fig. 2B), up-regulation of matrix-degrading enzymes by OA-associated catabolic factor such as IL-1 $\beta$ and HIF-2 $\alpha$ was significantly inhibited by Regnase-1 overexpression (Fig. 4) and potentiated by Regnase-1 knock-out in chondrocytes (Fig. 5). Therefore, it is likely that up-regulated Regnase- 1 in OA chondrocytes may suppresse cartilage destruction 
by forming negative feedback loop of catabolic signaling such as expression of matrix-degrading enzymes in chondrocytes.

\section{Identification and characterization of Regnase-1 targets in chondrocytes}

Regnase-1 directly binds to stem-loop structures in the 3'-UTRs of target mRNAs to cause their decay (11, 12). To identify target mRNAs of Regnase-1 in chondrocytes, we infected primary-culture chondrocytes with Ad-Regnase-1 or Ad-shRegnase-1 to up- and down-regulate Regnasse-1, respectively, and conducted microarray analyses. From among the 161 genes found to be down-regulated ( $<0.6$-fold) by Regnase- 1 overexpression, only nine were up-regulated ( $>1.5$-fold) by Regnase- 1 knockdown (Fig. 6A, Supplementary Table 3). In contrast, of the 67 genes that were up-regulated (>1.5-fold) by Regnase- 1 knockdown, 10 genes were down-regulated (<0.7-fold) by Regnase-1 overexpression (Fig. 6A, Supplementary Table 4). The genes that were up-regulated by Regnase- 1 overexpression or down-regulated by Regnase-1 knockdown are listed in Supplementary Tables 5 and 6, respectively.

Of the putative targets of Regnase-1, serum amyloid A3 (SAA3) was the most markedly up-regulated by Regnase-1 knockdown and down-regulated by Regnase-1 overexpression (Fig. 6B). SAA3 is a member of the SAA protein family of apolipoproteins. Different isoforms of SAA are expressed constitutively (constitutive SAAs) at different levels or in response to inflammatory stimuli (acute-phase SAAs) [26]. We first used the SAA3-3' - UTR reporter plasmid to test whether SAA3 was a bona fide direct target of Regnase-1. We found that the expression of WT Regnase- 1 reduced luciferase activity, whereas that of D141N Regnase-1, which lacks RNase activity [13], did not (Fig. 6C). In addition to SAA3, we also found that SAA1, SAA2, and SAA4 also tended to be up-regulated by Ad-shRegnase-1 and down-regulated by AdRegnase-1 (Fig. 6B, Supplementary Fig. 4). Among the SAA family members, SAA3 was the most abundant in chondrocytes (Fig. 6D) and the most significantly modulated by Regnase-1 expression (Supplementary Fig. 4). We therefore selected SAA3 as a Regnase- 1 target for functional analysis in OA pathogenesis.

\section{SAA3, a Regnase-1 target, does not modulate OA pathogenesis}

The function of SAA3 in OA pathogenesis was examined by its overexpression in joint tissues via IA injection of Ad-SAA3. Compared with Ad-C injection, IA injection of Ad-SAA3 in mice did not affect cartilage homeostasis or cause synovial inflammation (Fig. 6E). Consistently, overexpression of SAA3 in primary-culture chondrocytes did not modulate the mRNA levels of matrix-degrading enzymes (MMP3, MMP13, and ADAMTS5) or cartilage ECM molecules (type Il collagen and aggrecan) (Fig. 6F). Furthermore, IA injection of Ad-SAA3 in sham- or DMM-operated mice did not affect post-traumatic OA manifestations, such as cartilage destruction, osteophyte formation, and synovitis (Fig. 6G and H). Our results collectively indicate that SAA3 overexpression in joint tissues did not cause or modulate $\mathrm{OA}$ pathogenesis in mice, suggesting that the chondro-protective functions of Regnase- 1 in post-traumatic OA cartilage destruction is mediated by unidentified other target(s) of Regnase-1, rather than SAA3, in chondrocytes. 


\section{Discussion}

The post-transcriptional regulation of mRNA stability is an important checkpoint for gene expression, and ribonucleases play a central role in this process. Consequently, abnormalities in ribonucleases have been associated with multiple disease $[12,27,28]$. Indeed, we previously demonstrated that ZFP36L1, an RNAbinding protein that binds to AU-rich elements (AREs) in the $3^{\prime}$-UTRs of its target mRNAs, regulates OA pathogenesis by modulating the mRNA decay of HSP70 family members [29]. Here, we demonstrate that up-regulated Regnase- 1 in OA chondrocytes protects against post-traumatic OA cartilage destruction by modulating the expression levels of matrix-degrading enzymes. Therefore, our results indicate that upregulated Regnase-1 in OA chondrocytes forms a negative feedback loop to function as a chondroprotective effector molecule in OA cartilage destruction.

One of the key findings of our study is that Regnase- 1 inhibits the expression levels of matrix-degrading enzymes, such as MMP3, MMP13, and ADAMTS5, in chondrocytes, and thereby suppresses DMMinduced cartilage destruction. Regnase-1 overexpression suppresses the IL-1 $\beta$ - or HIF-2a-induced upregulations of MMP3, whereas KO of Regnase-1 potentiates the up-regulations of MMP3, MMP13, and ADAMTS5 with corresponding modulation of cartilage destruction. Regnase- 1 causes decay of target mRNAs by specifically binding to UAU and UGU loops of the 3'-UTR located more than 20 nucleotides away from the stop codon [30]. According to RegRNA2.0 analysis [31], the mRNAs of mouse MMP3, MMP13, and ADAMTS5 have loops that meet these criteria. However, unlike the regulation of these enzymes in the presence of catabolic stimuli (i.e., IL-1 $\beta$ and HIF-2a), overexpression or knockdown of Regnase-1 in unstimulated chondrocytes did not markedly modulate the expression levels of these enzymes (Supplementary Tables 3-6). Therefore, our results indicate that Regnase-1 regulates the expression levels of matrix-degrading enzymes via indirect regulatory mechanisms, rather than direct targets of Regnase- 1.

Regnase-1 regulates a variety of different target mRNAs in different cell types [11-15]. The mRNA of IL-6 has been identified as a Regnase-1 target in multiple cell types, including macrophages [20,32], T cells $[13,14]$, and chondrocytes [16]. In unstimulated human OA chondrocytes, overexpression of Regnase-1 decreases the IL- 6 mRNA level whereas expression of D141N mutant Reganse-1 (lacking RNase activity) does not [16]; this suggested that the IL-6 mRNA is a target of Reganse-1, although this was not confirmed in the previous study. Because IL-6 plays a critical role in OA pathogenesis [19], we initially speculated that Regnase- 1 may exert its effects by modulating the IL- 6 mRNA. However, similar to our observations regarding matrix-degrading enzymes, we found that overexpression or knockdown of Regnase- 1 in unstimulated chondrocytes did not modulate the mRNA expression of IL-6 (Supplementary Tables 3-6). This suggests that IL-6 mRNA is not a direct target of Regnase-1 in mouse articular chondrocytes and does not play a role in the ability of Regnase- 1 to regulate post-traumatic OA cartilage destruction. This is consistent with the fact that Regnase- 1 targets vary dramatically among different cells and conditions $[11,33]$. It has been suggested that the process of Regnase- 1 recognition is likely to be determined by multiple factors and distinct layers of regulation that may differ among cell types [11, 33]. 
To identify mediator(s) through which Regase-1 regulates OA cartilage destruction, we tried to identified Regnase-1 target mRNAs via microarray analysis in chondrocytes with overexpression or knockdown of Regnase- 1 . We found that SAA family members met the criteria for Regnase- 1 targets: they were downregulated by Regnase- 1 overexpression and up-regulated by Regenase- 1 knockdown, and contain UAU and UGU loops in their 3'-UTRs. SAA1 and SAA2 are classic acute phase response serum proteins in humans and mice; SAA3 is a pseudogene in humans that is transcribed but not translated; and SAA4 is expressed constitutively in humans [26]. The SAAs have been suggested to play roles in inflammatory diseases, such as atherosclerosis, rheumatoid arthritis, and chronic inflammatory bowel disease, and may function in cholesterol transport [34]. SAAs have also been reported to induce the expression of proinflammatory genes via NF-KB pathways [35], and recombinant SAA3 was reported to induce MMP13 expression in rabbit articular chondrocytes [36]. Here, we characterized the possible functions of SAA3 in

the Regnase- 1 regulation of OA pathogenesis, as it was the most abundantly expressed and significantly modulated SAA isoform in our system. However, our results revealed that overexpression of SAA3 in mouse articular chondrocytes does not modulate the expression of matrix-degrading enzymes or cartilage ECM molecules, and OA cartilage destruction is not modulated by the overexpression of SAA3 in mouse joint tissues. Therefore, it is likely that unidentified Regnase- 1 targets other than SAA3 may mediate the chondro-protective functions of Regnase-1 during OA pathogenesis.

\section{Conclusions}

The present results suggest that up-regulated Regnase-1 in OA chondrocytes may functions as a chondro-protective effector molecule during OA pathogenesis by forming negative feedback loop of catabolic signaling such as expression of matrix-degrading enzymes in OA chondrocytes.

\section{Abbreviations}

ARE: AU-rich elements; ECM: extracellular matrix; IL: interleukin; HIF: hypoxia-inducible factor; KO: knockout; MMP: matrix metalloproteinase; OA: osteoarthritis; SAA: serum amyloid A; WT: wild-type

\section{Declarations}

\section{Funding}

This work was supported by grants from the National Research Foundation of Korea (2016R1A3B1906090, 2016R1A5A1007318, and 2019M3A9A8065758) and the Korea Health Industry Development Institute (H114C3484).

\section{Availability of data and materials}

The data supporting the conclusions of this article are included within the article.

\section{Ethics approval and consent to participate}


Not applicable

\section{Competing interests}

The authors declare that they have no competing interests.

\section{Consent for publication}

Not applicable

\section{Authors' contributions}

JIY: study design, data acquisition, data analysis, data interpretation, manuscript preparation, and manuscript approval. JSC (jschun@gist.ac.kr) takes responsibility for the integrity of this work.

\section{References}

1. Martel-Pelletier J, Barr AJ, Cicuttini FM, Conaghan G, Cooper C, Goldring MB, Goldring SR, Jones G, Teichtahl AJ, Pelletier JP. Osteoarthritis. Nat Rev Dis Primers 2016;2:16072.

2. Mehana EE, Khafaga AF, El-Blehi SS. The role of matrix metalloproteinases in osteoarthritis pathogenesis: an updated review. Life Sci 2019;234:116786.

3. Blom AB, van Lent PL, Libregts $S$, Holthuysen $A E$, van der Kraan PM, van Rooijen N, van den Berg WB. Crucial role of macrophages in matrix metalloproteinase-mediated cartilage destruction during experimental osteoarthritis: involvement of matrix metalloproteinase 3. Arthritis Rheum 2007;56:14757.

4. Little CB, Barai A, Burkhardt D, Smith SM, Fosang AJ, Werb Z, Shah M, Thompson EW. Matrix metalloproteinase 13-deficient mice are resistant to osteoarthritic cartilage erosion but not chondrocyte hypertrophy or osteophyte development. Arthritis Rheum 2009;60:3723-33.

5. Glasson SS, Askew R, Sheppard B, Carito B, Blanchet T, Ma HL, Flannery CR, Peluso D, Kanki K, Yang Z, Majumdar MK, Morris EA. Deletion of active ADAMTS5 prevents cartilage degradation in a murine model of osteoarthritis. Nature 2005;434:644-7.

6. Wojdasiewicz P, Poniatowski LA, Szukiewicz D. The role of inflammatory and anti-inflammatory cytokines in the pathogenesis of osteoarthritis. Mediators Inflamm 2014;2014:561459.

7. Yang S, Kim J, Ryu JH, Oh H, Chun CH, Kim BJ, Min BH, Chun JS. Hypoxia-inducible factor-2a is a catabolic regulator of osteoarthritic cartilage destruction. Nat Med 2010;16:687-93.

8. Kim JH, Jeon J, Shin M, Won Y, Lee M, Kwak JS, Lee G, Rhee J, Ryu JH, Chun CH, et al. Regulation of the catabolic cascade in osteoarthritis by the zinc-ZIP8-MTF1 axis. Cell 2014;156:730-43.

9. Choi WS, Lee G, Song WH, Koh JT, Yang J, Kwak JS, Kim HE, Kim SK, Son YO, Nam H, et al. The $\mathrm{CH} 25 \mathrm{H}-\mathrm{CYP7B1-RORa}$ axis of cholesterol metabolism regulates osteoarthritis. Nature 2019;566:2548. 
10. Labno A, Tomecki R, Dziembowski A. Cytoplasmic RNA decay pathways - enzymes and mechanisms. Biochim Biphys Acta 2016;1863:3125-47.

11. Mao R, Yang R, Chen X, Harhaj EW, Wang X, Fan Y. Regnase-1, a rapid response ribonuclease regulating inflammation and stress responses. Cell Mol Immunol. 2017;14:412-22.

12. Takeuchi O. Endonuclease Regnase-1/monocyte chemotactic protein-1-induced protein-1 (MCPIP1) in controlling immune responses and beyond. WIREs RNA 2018;9:e1449.

13. Uehata T, Iwasaki H, Vandenbon A, Mathushita K, Hernandez-Cuellar E, Kuniyoshi K, Satoh T, Mino T, Suzuki Y, Standley DM, et al. Malt1-induced cleavage of regnase-1 in CD4(+) helper T cells regulates immune activation. Cell 2013;153:1036-1049.

14. Wei J, Long L, Zheng W, Dhungana Y, Lim SA, Guy C, Wang Y, Qian C, Xu B, Kc A, et al. Targeting Regnase-1 programs long-lived effector T cells for cancer therapy. Nature 2019;576:471-6.

15. Lu W, Ning H, Gu L, Peng H, Wang Q, Fu M, Hoft DF, Liu J. MCPIP1 selectively destabilizes transcripts associated with an antiapoptotic gene expression program in breast cancer cells that can elicit complete tumor regression. Cancer Res 2016;76:1429-40.

16. Makki MS, Haseeb A, Haqqi TM. MicroRNA-9 promotion of interleukin-6 expression by inhibiting monocyte chemoattractant protein-induced protein 1 expression in interleukin-1 $\beta$-stimulated human chondrocytes. Arthritis Rheumatol 2015;67:2117-28.

17. Makki MS, Haqqi TM. Histone deacetylase inhibitor Vorinostat (SAHA, MK0683) perturb miR-9MCPIP1 axis to block IL-1 $\beta$-induced IL-6 expression in human OA chondrocytes. Connect Tissue Res 2017;58:64-75.

18. Jones SA, Jenkins BJ. Recent insights into targeting the IL-6 cytokine family in inflammatory diseases and cancer. Nat Rev Immunol 2018;18:773-89.

19. Ryu JH, Yang S, Shin Y, Rhee J, Chun CH, Chun JS. Interleukin-6 plays an essential role in hypoxiainducible factor $2 a$-induced experimental osteoarthritic cartilage destruction in mice. Arthritis Rheum 2011;63:2732-43.

20. Matsushita K, Takeuchi O, Standley DM, Kumagai Y, Kawagoe T, Miyake T, Satoh T, Kato H, Tsujimura T, Nakamura H, et al. Zc3h12a is an RNase essential for controlling immune responses by regulating mRNA decay. Nature 2009;458:1185-90.

21. Glasson SS, Blanchet TJ, Morris EA. The surgical destabilization of the medial meniscus (DMM) model of osteoarthritis in the 129/SvEv mouse. Osteoarthritis Cartilage 2007;15:1061-9.

22. Glasson SS, Chambers MG, van den Berg WG, Little CB. The OARSI histopathology initiative recommendations for histological assessments of osteoarthritis in the mouse. Osteoarthritis Cartilage 2010;18,Suppl 3:S17-23.

23. Krenn V, Morawietz L, Burmester GR, Kinne RW, Mueller-Ladner U, Muller B, Haupl T. Synovitis score: discrimination between chronic low-grade and high grade synovitis. Histopathology 2006;49:35864.

24. Das Neves Borges P, Vincent TL, Marenzana M. Automated assessment of bone changes in crosssectional micro-CT studies of murine experimental osteoarthritis. Plos One 2017;12: e0174294. 
25. Gosset M, Berenbaum, F, Thiron S, Jacques C. Primary culture and phenotyping of murine chondrocytes. Nat Proc 2008;3:1253-60.

26. Sack GH. Serum amyloid A - a review. Mol Med 2018;24:46.

27. Canestrari E, Paroo Z. Ribonuclease as drug targets. Trend Phamacol Sci 2018;39:855-66.

28. Mattijssen S, Welting TJM, Pruijin GJM. RNase MRP and disease. WIREs RNA 2010;1:102-16.

29. Son YO, Kim HE, Choi WS, Chun CH, Chun JS. RNA-binding protein ZFP36L1 regulates osteoarthritis by modulating members of the heat shock protein 70 family. Nat Commun 2019;10:77.

30. Mino T, Murakawa Y, Fukao A, Vendenbon A, Wessels HH, Ori D, Uehata T, Tartey S, Akira S, Suzuki Y, et al. Regnase- 1 and roquin regulate a common element in inflammatory mRNAs by spatiotemporally distinct mechanisms. Cell 2015;161:1058-73.

31. Chang TH, Huang HY, Hsu JBK, Weng SL, Horng JT, Huang HD. An enhanced computational platform for investigating the roles of regulatory RNA and for identifying functional RNA motifs. BMC Bioinformatics 2013;14:S4.

32. Liang J, Wang J, Azfer A, Song W, Tromp G, Kolattukudy PE, Fu M. A novel CCCH-zinc finger protein family regulates proinflammatory activation of macrophages. J Biol Chem 2008;283:6337-46.

33. Kidoya H, Muramatsu F, Shimamura T, Jia W, Satoh T, Hayashi Y, Naito H, Kunisaki Y, Arai F, Seki M, et al. Regnase-1-mediated post-transcriptional regulation is essential for hematopoietic stem and progenitor cell homeostasis. Nat Commun 2019;10:1072.

34. Thompson JC, Wilson PG, Shridas P, Ji A, de Beer M, de Beer FC, Webb NR, Tannock LR. Serum amyloid A3 is pro-atherogenic. Atherosclerosis. 2018;268:32-5.

35. Jijon HB, Maddsen KL, Walker JW, Allard B, Jobin C. Serum amyloid A activates NF-kappaB and proinflammatory gene expression in human and murine intestinal epithelial cells. Eur $\mathrm{J}$ Immunol 2005;35:718-26.

36. Vallon R, Freuler F, Desta-Tsedu, Robeva A, Dawson J, Wenner P, Engelhardt P, Boes L, Schnyder J, Tschopp C, et al. Serum amyloid A (apoSAA) expression is up-regulated in rheumatoid arthritis and induces transcription of matrix metalloproteinases. J Immunol 2001;166:2801-7.

\section{Figures}


A

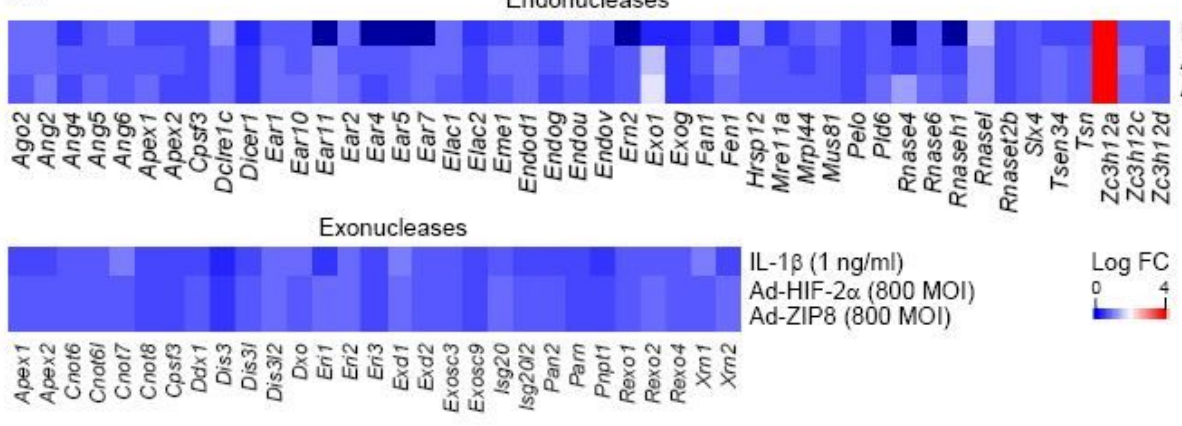

C

IL-1B (1 ng/ml) Ad-HIF- $2 \alpha$ ( $800 \mathrm{MOI})$ Ad-ZIP8 (800 MOI)

(1)



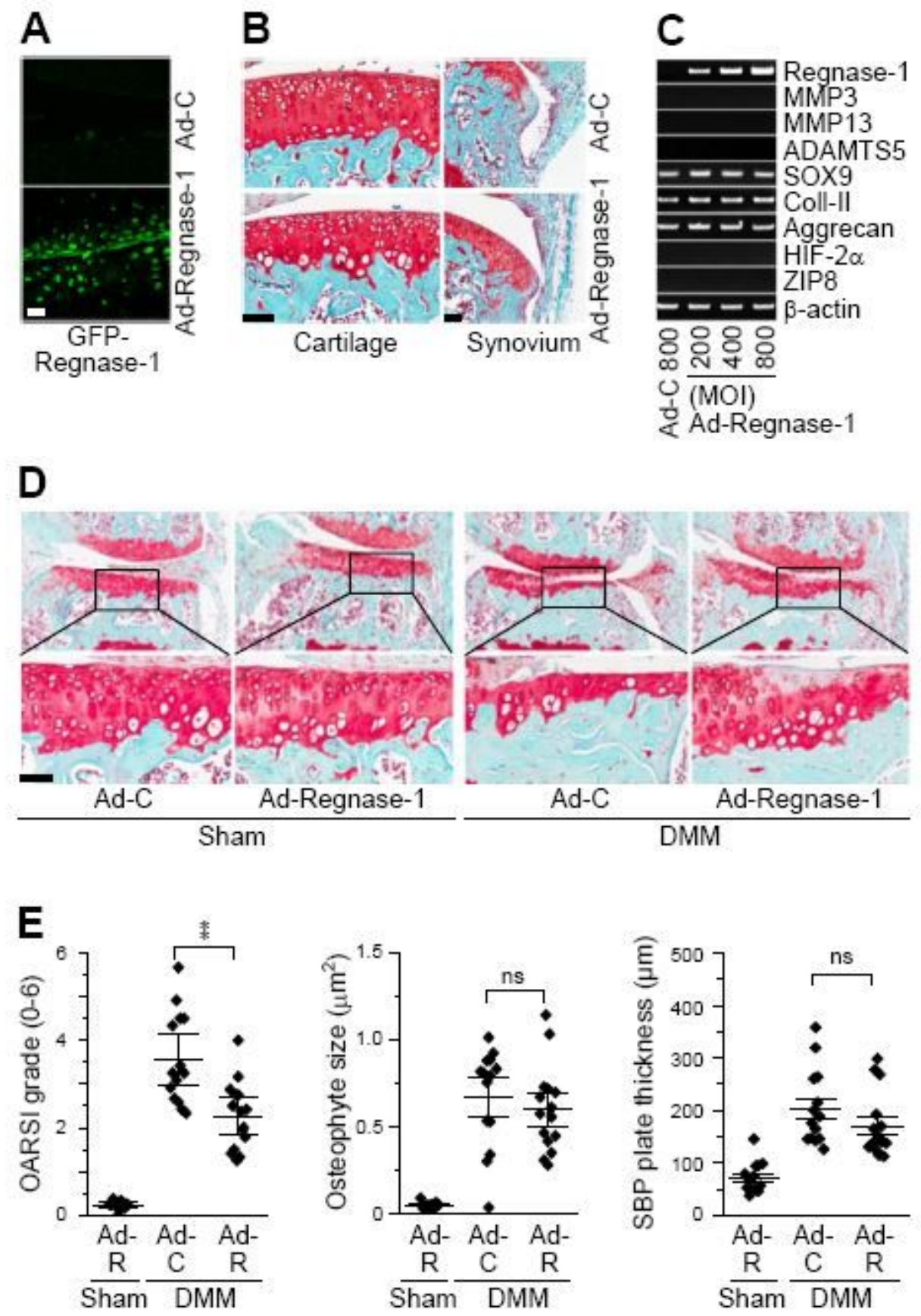

\section{Figure 2}

Overexpression of Regnase- 1 in joint tissue ameliorates post-traumatic OA cartilage destruction in mice. A GFP fluorescence image in knee joint cartilage of mice IA-injected with Ad-C or GFP-tagged Ad-Regnase$1(n=5)$. B Representative joint-section images of WT mice IA-injected with empty virus (Ad-C; $n=5$ mice) or Ad-Regnase-1 ( $n=8$ mice). C Representative RT-PCR images of the indicated molecules in chondrocytes treated with the indicated MOI of Ad-C or Ad-Regnase- $1(n=5)$. D and E Sham- or DMMoperated WT mice were IA-injected with Ad-C or Ad-Regnase-1 (Ad-R). Safranin-O staining images (D) and 
scoring of OARSI grade, osteophyte size, and subchondral bone plate (SBP) thickness (E) at 8 weeks after operation ( $n=15$ mice per group). Means $\pm 95 \% \mathrm{Cl}$ with Mann-Whitney $\mathrm{U}$ test for OARSI grade and means \pm s.e.m. with two-tailed t-test for osteophyte size and SBP thickness. ${ }^{*} p<<0.005$. ns, not significant. Scale bar: $50 \mu \mathrm{m}$.
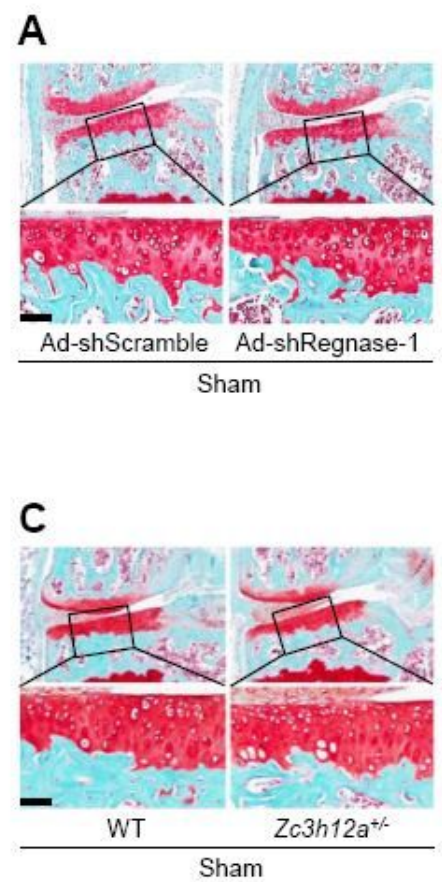
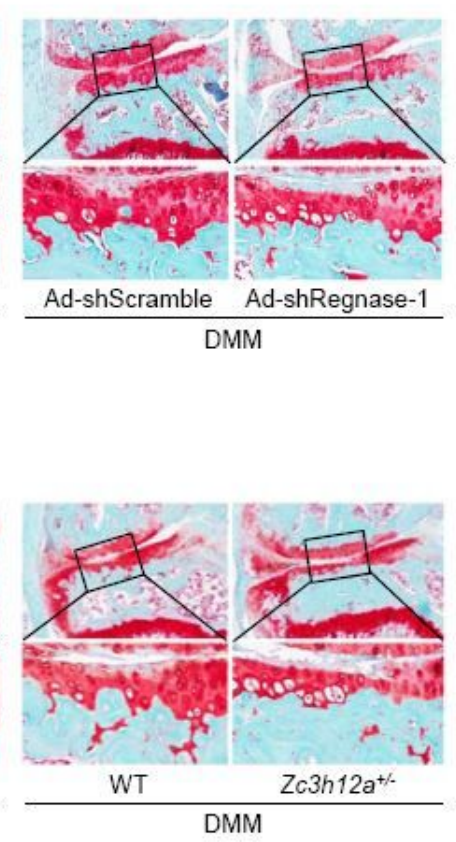
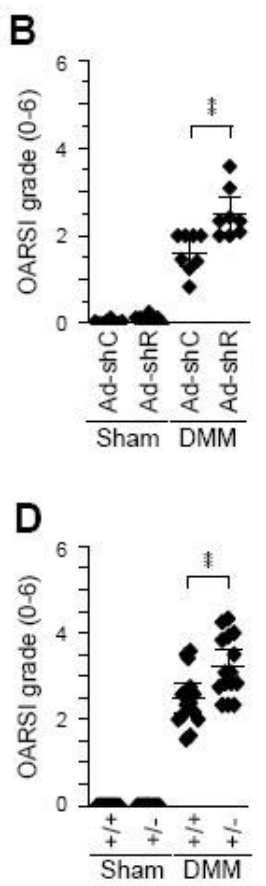
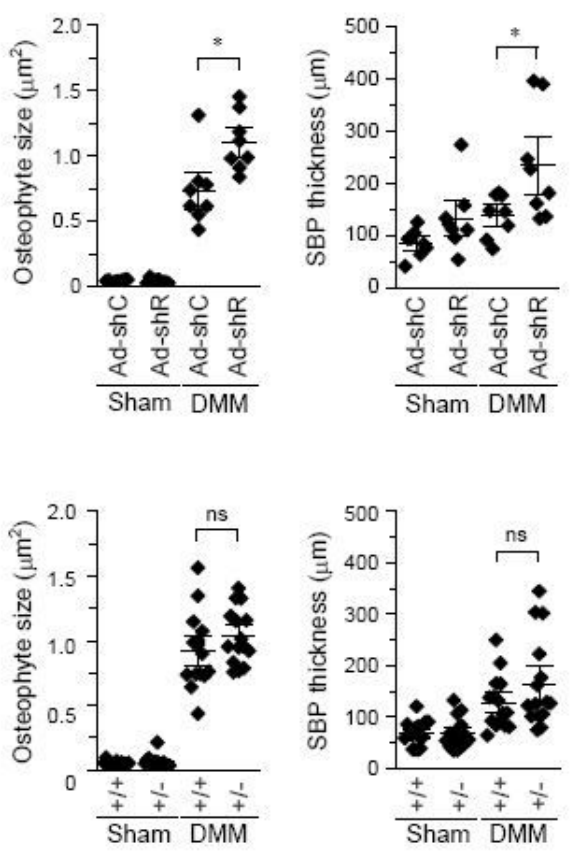

\section{Figure 3}

Knockdown or genetic ablation of Regnase-1 exacerbates OA pathogenesis in mice. A and B Sham- or DMM-operated WT mice were IA-injected with Ad-shScramble (Ad-shC) or Ad-shRegnase-1 (Ad-shR) and sacrificed at 6 weeks after operation ( $n=8$ mice per group). Safranin-0 staining images $(A)$ and scoring of OARSI grade, osteophyte size, and SBP thickness (B). C and D WT (Zc3h12a+/+) and Zc3h12a+/mice were subjected to sham operation or DMM surgery and sacrificed at 6 weeks after operation $(n=15$ mice per group). Representative Safranin-O staining images (C) and scoring of OARSI grade, osteophyte size, and SBP thickness (D). Means $\pm 95 \% \mathrm{Cl}$ with Mann-Whitney $\mathrm{U}$ test for OARSI grade and means \pm s.e.m. with two-tailed t-test for osteophyte size and SBP thickness. ${ }^{*} p<0.05$ and ${ }^{* \star} p<0.005$. ns, not significant. Scale bar: $50 \mu \mathrm{m}$. 
A
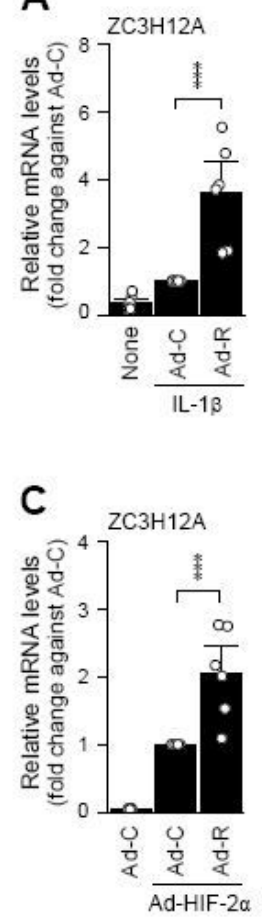

B
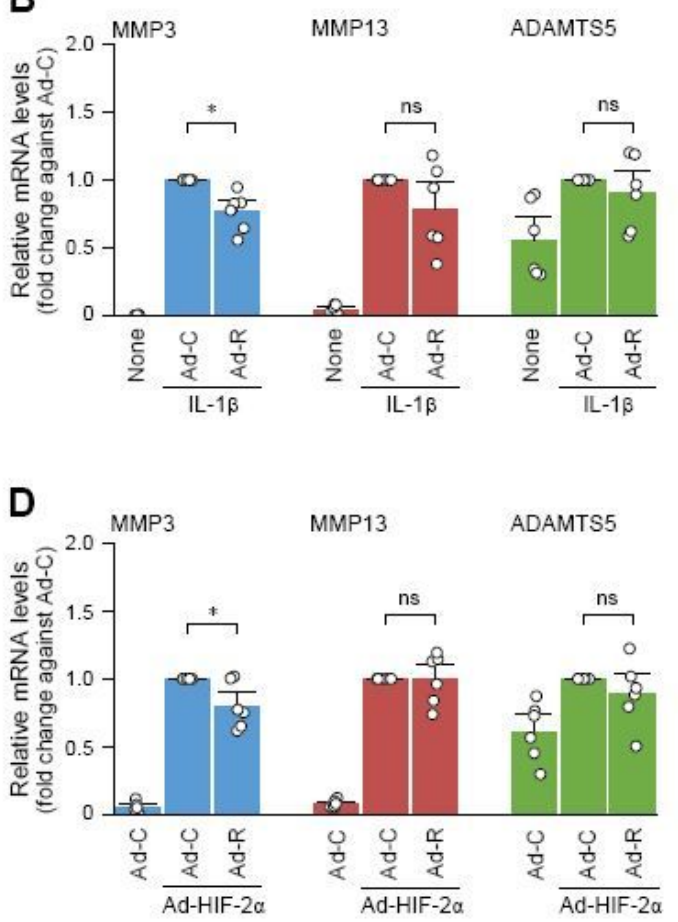
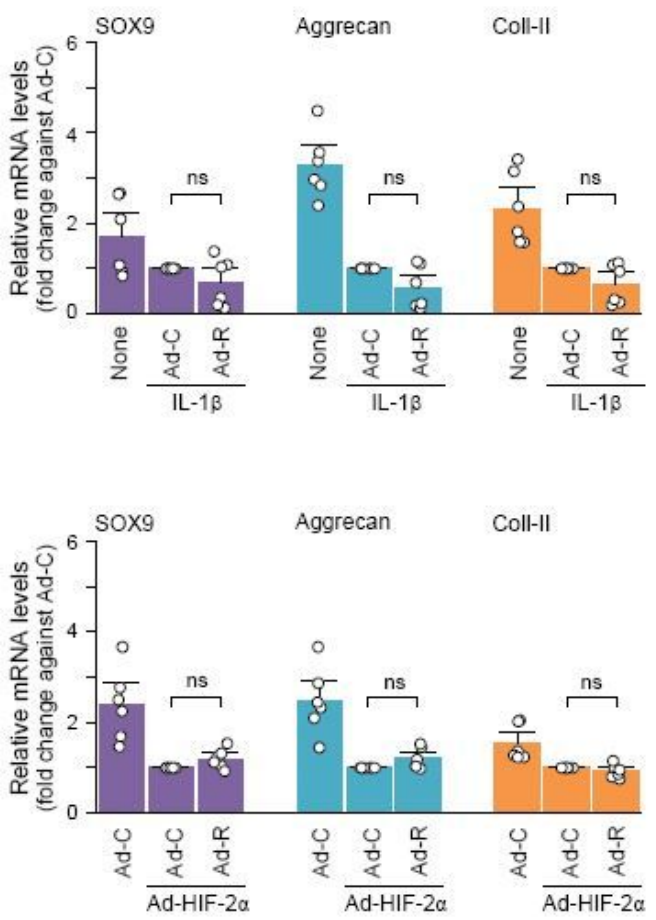

\section{Figure 4}

Effects of Regnase-1 overexpression on the expression of matrix-degrading enzymes and ECM molecules. A and B Primary-culture chondrocytes were infected with $800 \mathrm{MOI}$ of Ad-C or Ad-Regnase-1 (Ad-R) for 12 hours, and exposed to IL-1》 $(0.01 \mathrm{ng} / \mathrm{ml})$ for an additional 24 hours. The indicated molecules were quantified by qRT-PCR analysis $(n=6)$. C and D Primary-culture chondrocytes were preinfected with $400 \mathrm{MOI}$ of Ad-C or Ad-Regnase-1 (Ad-R) for 12 hours, and additionally infected with 400 $\mathrm{MOI}$ of Ad-HIF-2a for 24 hours. Untreated chondrocytes were infected with $800 \mathrm{MOI}$ of Ad-C as a control. The indicated molecules were quantified by qRT-PCR analysis $(n=6)$. Means \pm s.e.m. with one-way ANOVA with Bonferroni's post hoc test. ${ }^{*} p<0.05$ and ${ }^{* *} \mathrm{p}<0.0005$. ns, not significant. 

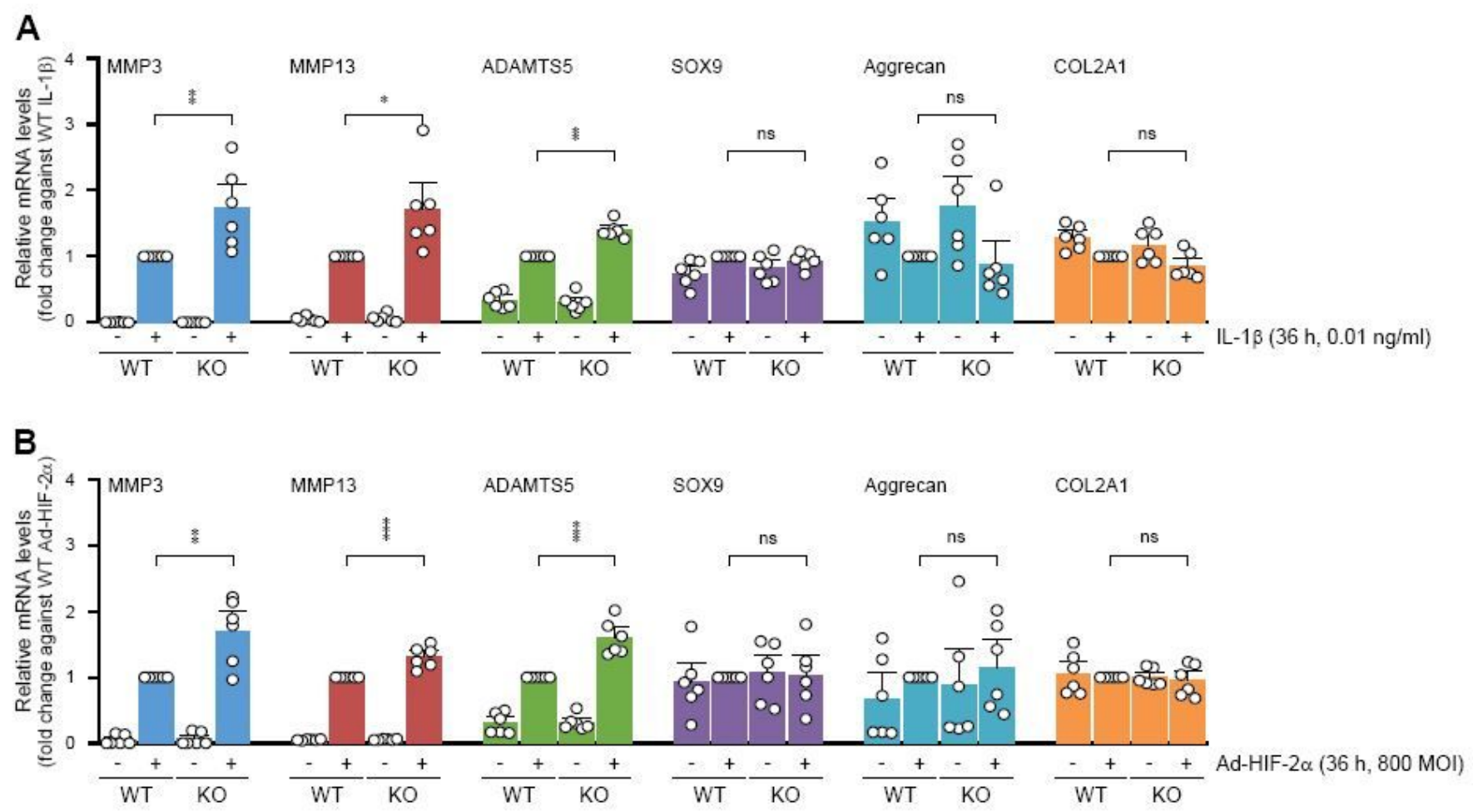

\section{Figure 5}

Zc3h12a-/- chondrocytes exhibit enhanced expression of matrix-degrading enzymes when treated with IL$1 \rrbracket$ or Ad-HIF-2a. A and B WT and KO (Zc3h12a-/-) chondrocytes were treated with $0.01 \mathrm{ng} / \mathrm{ml}$ of IL-1区 (A) or infected with $800 \mathrm{MOI}$ of Ad-HIF-2a (B) for 36 hours. mRNA levels of the indicated molecules were determined by qRT-PCR analysis $(n=6)$. Values are means \pm s.e.m. with one-way ANOVA with Bonferroni test. ${ }^{*} p<0.05,{ }^{\star \star} p<0.005,{ }^{\star \star \star} p<0.0005$. ns, not significant. 
A

\begin{tabular}{lc}
\hline Treatment & No \\
\hline Down-regulated genes & 161 \\
by Regnase-1 (<0.6 fold) & \\
Upregulated by Regnase-1 knockdown & 9 \\
Not affected & 154 \\
Downregulated by shRegnase-1 & 0 \\
\hline Up-regulated genes & 67 \\
by shRegnase-1 ( $>1.5$ fold) & \\
Upregulated by Regnase-1 overexpression 10 \\
Not affected & 48 \\
Downregulated by Regnase-1 (<0.7 fold) & 10 \\
\hline
\end{tabular}

B

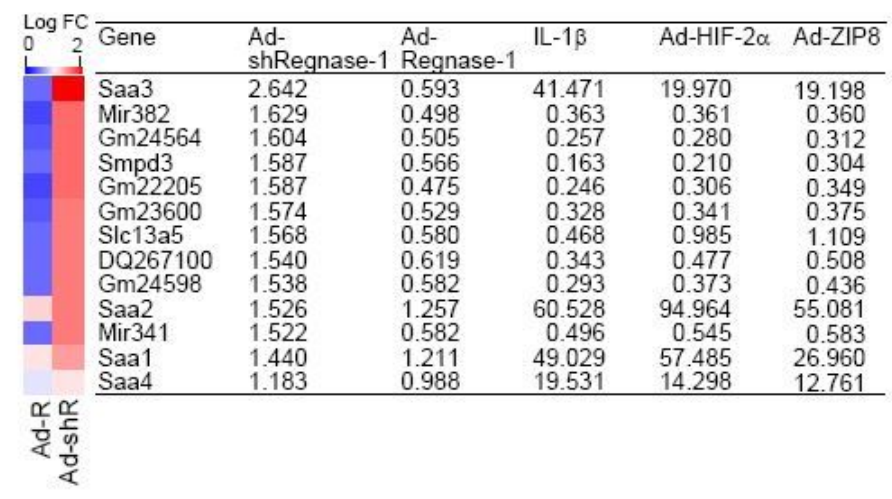

$\mathbf{E}$
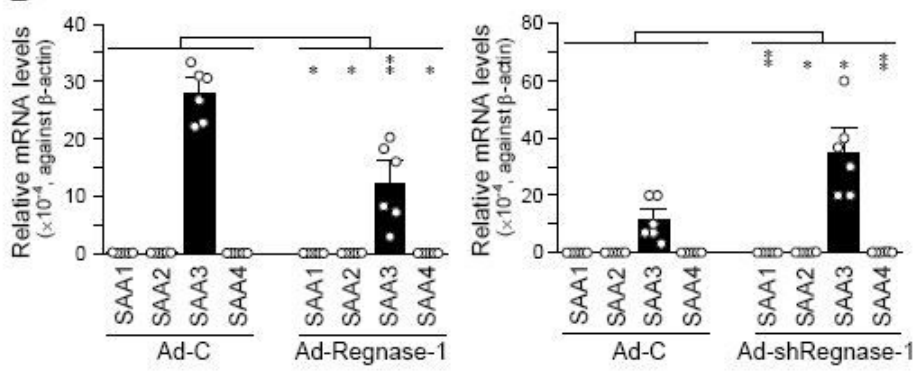

C

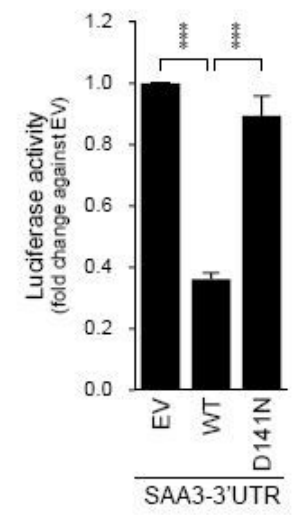

$\mathbf{F}$

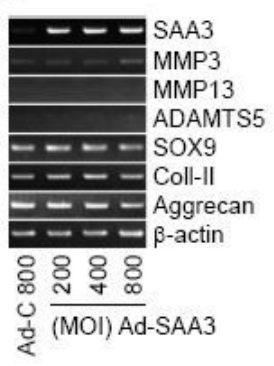

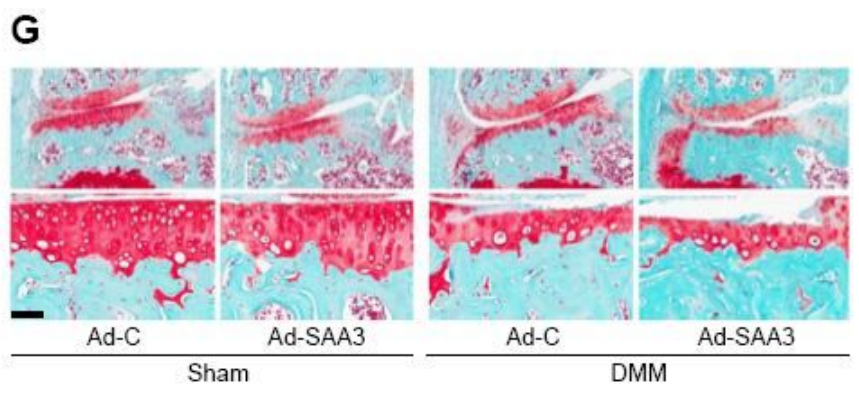
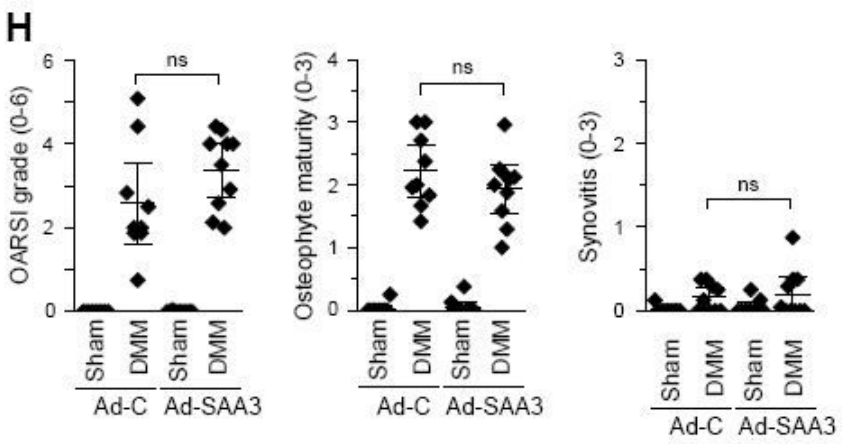

Figure 6

SAA3, a target of Regnase-1, does not modulate OA pathogenesis. A and B Number of genes modulated by Regnase-1 (A) and list of the top 13 genes that were most markedly regulated by Ad-shRegnase-1 (B). C Luciferase assay $(n=4)$ in chondrocytes transfected with a reporter plasmid containing the SAA3 3'UTR sequence. Chondrocytes were co-transfected with empty vector (EV), WT Regnase-1 (WT), or D141N Regnase-1 (D141N). D qRT-PCR analysis $(n=6)$ of SAA family members in chondrocytes infected with Ad-Regnase- 1 or Ad-shRegnase-1 for 36 hours. Means \pm s.e.m. with two-tailed t-test. E Safranin-0 staining images in WT mice IA-injected with Ad-C or Ad-SAA3 ( $n=10$ mice per group). Mice were sacrificed at 3 weeks post-injection. $F$ Representative RT-PCR images of the indicated molecules in chondrocytes infected with Ad-C or Ad-SAA3 for 36 hours $(n=6)$. G and H Sham- or DMM-operated WT mice were IAinjected with Ad-C or Ad-SAA3. Safranin-0 staining images $(G)$ and scoring of OARSI grade, osteophyte size, and SBP thickness $(H)$ at 6 weeks after the operation ( $n=10$ mice per group). Means $\pm 95 \% \mathrm{Cl}$ with Mann-Whitney $\mathrm{U}$ test for OARSI grade and means \pm s.e.m. with two-tailed t-test for osteophyte size and SBP thickness. ${ }^{*} p<0.05$. ns, not significant. Scale bar: $50 \mu \mathrm{m}$. 


\section{Supplementary Files}

This is a list of supplementary files associated with this preprint. Click to download.

- SupplementaryMaterials.pdf 